\title{
¿Qué son los modelos físicos?
}

\author{
José Luis Rolleri \\ Universidad Autónoma de Querétaro
}

\section{Resumen}

En este artículo analizamos los principales conceptos propuestos de modelo con el propósito de resaltar el carácter idealizado y abstracto de los modelos físicos, que generalmente es pasado por alto en la literatura filosófica relevante desde diversas posiciones, como el instrumentalismo y el pragmatismo; el cual, sin embargo tiene importantes implicaciones en problemas filosóficos como el del realismo científico. Desde una posición conceptualista, sostenemos que los modelos desidealizados son los vehículos para conceptualizar los sistemas físicos desde la perspectiva de una teoría.

Palabras clave: idealización, abstracción, aproximación, desidealización, concretización.

\section{Abstract}

In this paper we analyze the main intended concepts of model in order to underline the idealized and abstract character of physical models, that generally is overpass in the relevant philosophical literature from diverse positions on the scientific knowledge, such as instrumentalism and pragmatism, which however has important implications on philosophical problems such as that of scientific realism. From a conceptualistic position we hold that the de-idealized models are the vehicles to conceptualized physical systems from the perspective of a theory. 
Keywords: Idealization, Abstraction, Approximation, De-idealization, Concretization.

\section{Introducción}

En las últimas tres décadas el concepto de modelo se ha convertido en un concepto central en los estudios, discusiones y tesis filosóficos sobre la ciencia; sin embargo, en lugar de lograrse un consenso sobre qué son los modelos científicos, ha proliferado una diversidad de propuestas sobre los modelos en ciencia — desde diversas posiciones y con diferentes propósitos- que, si bien enriquece el estudio sobre éstos, entorpece las discusiones y la comprensión de las tesis filosóficas. ${ }^{1}$

El concepto de modelo desplazó al de teoría en estas décadas (al concepto de teoría del empirismo lógico y el racionalismo crítico como un sistema nomológico deductivo, que dominó el escenario de la filosofía de la ciencia desde la década de los años 30 hasta la de los 70 del pasado siglo), planteándose entonces la cuestión sobre la relación entre las teorías científicas y los modelos. Con respecto a lo anterior, podemos señalar dos tesis que se encuentran en los extremos: por un lado, los filósofos estructuralistas, como Joseph Sneed, Ulises Moulines y Wolfgang Balzer, mantienen que las teorías son redes jerarquizadas de clases de modelos (véase Balzer, Moulines y Sneed, 1987); por el otro, Nancy Cartwright, Margaret Morrison y Mary Morgan sostienen que los modelos son relativamente independientes o autónomos de las teorías (véase Cartwright, 1983 y Morrison y Morgan, 1999). Sin embargo, unos y

${ }^{1}$ La diversidad de modelos propuestos abarca modelos matemáticos, materiales, analógicos, interpretativos, representativos, idealizados y ficticios, entre otros. Al respecto, véase el artículo "Models in Science" (2006) de Roman Frigg y Stephan Hartmann en la Stanford Encyclopedia of Philosophy (en línea). 
otras conciben a los modelos de maneras distintas. Los primeros adoptan el concepto lógico de modelo; a saber, el de una estructura que satisface las leyes de una teoría. Las segundas toman por modelo casi todo lo que los científicos consideran como tal, o llaman así, sin ofrecer una caracterización general de los modelos.

Como el concepto lógico de modelo es un concepto claro y preciso, los estructuralistas logran ofrecer una concepción de las teorías a la vez clara y precisa, desde un punto de vista formal. No obstante, al definir a los modelos antes que nada como estructuras conjuntistas abstractas, les surge el problema de cómo se conectan ese tipo de estructuras con el mundo físico (véase Muller, 2011). Considerando que los modelos son entidades autónomas de las teorías, Cartwright, Morrison y Morgan mantienen que hacen las veces de agentes intermediarios entre las teorías, por un lado, y los fenómenos del mundo físico, por el otro. Sin embargo, no consiguen aclarar la cuestión involucrada sobre la conexión entre los modelos y los fenómenos - al menos, Cartwright no lo logra con su noción de modelo representativo, como indicamos adelante.

El objetivo de este artículo es examinar los principales conceptos de modelo propuestos para resaltar algunos rasgos propios de los modelos en física, principalmente su carácter idealizado y abstracto, que se ha soslayado en aras de sostener tesis sobre el conocimiento científico desde posiciones filosóficas, como la instrumentalista o pragmatista, entre otras.

\section{El concepto lógico de modelo}

Durante sus investigaciones en metamatemática, Alfred Tarski elaboró una teoría de modelos para estudiar ciertas propiedades de las teorías formales, lógicas o matemáticas. Con referencia a ellas, Tarski definió que: "Una realización posible en la que todos los enunciados válidos de una teoría $T$ son satisfechos se llama un 
modelo de T’ (1953: 11), donde por una realización posible se entiende una estructura matemática de tipo lógico apropiado.

En la década de 1950, Patrick Suppes y sus colegas trasladaron este concepto lógico de modelo al campo de las ciencias empíricas, sociales y naturales, sosteniendo que el significado del concepto de modelo es el mismo en matemáticas y en las ciencias empíricas, mientras que la diferencia que hay en esas disciplinas reside en los usos de ese concepto (Cfr. Suppes, 1960: 112).

El propio Suppes, a su vez, propuso que las axiomatizaciones de las teorías científicas - principal, pero no exclusivamente, las teorías físicas - deben llevarse a cabo usando la teoría intuitiva de conjuntos, en oposición a las axiomatizaciones en los lenguajes formalizados de la lógica (Cfr., Suppes, 1953).

Si tomamos en cuenta que, en la anterior definición de modelo de Tarski, los enunciados válidos de una teoría son los axiomas de la teoría y sus consecuencias lógicas (teoremas), entonces, bajo la propuesta suppesiana, los modelos de las teorías físicas resultan ser estructuras conjuntistas que satisfacen las leyes de la teoría postuladas como axiomas. Se tiene, entonces, que los modelos físicos son modelos conjuntistas.

Ante esto, Suppes agrega que las axiomatizaciones conjuntistas de las teorías deben ser complementadas con las interpretaciones físicas propuestas (intended) de sus conceptos y leyes. Por ejemplo, con respecto a una axiomatización conjuntista de la mecánica clásica de partículas, que contiene como dominio un conjunto $\mathrm{P}$ de partículas clásicas, ${ }^{2}$ Suppes anota que:

Es suficientemente simple ver cómo un modelo físico real en el sentido de los físicos de la mecánica clásica de partículas está relacionado a este sentido conjuntista de modelo. Simplemente podemos tomar el conjunto de partículas como, en el caso del

${ }^{2}$ En el capítulo 12 de Suppes (1957) se incluye tal axiomatización. 
sistema solar, el conjunto de los cuerpos planetarios [...] Podría parecer que no hay ningún caso en argüir acerca de cuál uso de la palabra 'modelo' es primario o más apropiado en las ciencias empíricas. Mi opinión es que el uso conjuntista es el más fundamental (1960: 114).

Estas propuestas han tenido una repercusión que es difícil sobreestimar. Además de las axiomatizaciones conjuntistas que realizaron Suppes y colaboradores, han sido la semilla del programa estructuralista en filosofía de la ciencia, iniciado por Sneed en (1971), dentro de la corriente actual que se conoce como la concepción semántica o modelística de la ciencia. ${ }^{3}$

Empero, lo que nos interesa destacar aquí es la tesis de Suppes de que el concepto lógico de modelo es apropiado para el estudio de los modelos en las ciencias físicas. Algunos destacados autores que adoptan la llamada concepción semántica de las teorías científicas, como Bas van Fraassen (1980) y Ronald Giere (1988), sólo hacen comentarios a favor sobre su posible adecuación, sin asumirla estrictamente. Otros importantes autores que han escrito sobre los modelos científicos, como Michael Redhead (1980), R. I. G. Hughes (1997) y Chuang Liu (2004), no la toman en cuenta. F. A. Muller (2011) hace indirectamente un fuerte cuestionamiento a la plausibilidad de esa tesis suppesiana, al mostrar que los enfoques formales, en particular el enfoque conjuntista de Suppes, precisamente por su carácter puramente formal, matemático, generan ciertos problemas como la pérdida de contenido y referencia factual, puesto que las entidades que efectivamente cumplen las leyes

${ }^{3}$ En referencia a las teorías científicas reconstruidas desde la metateoría estructuralista de la ciencia, Moulines anota que "En el momento que escribo estas líneas, al menos unas cincuenta teorías de distintas disciplinas científicas - de la física a la sociología, pasando por la química, la biología, la psicología y la economía- han sido reconstruidas con todos los detalles y la precisión necesaria" (2008: 136). 
de las teorías físicas axiomatizadas a la Suppes, son estrictamente hablando estructuras abstractas, en contraste con entidades físicas concretas, como los planetas de nuestro Sistema Solar. En otras palabras, los modelos conjuntistas son entidades abstractas desprovistas tanto de contenido factual como de referencia a los sistemas físicos a los que se pretenden aplicar.

\section{Los conceptos de modelo en la ciencia}

Los científicos usan el término modelo de manera polisémica y equívoca. Comparando ciertos usos de ese término con el sentido lógico de modelo, Suppes observa que es una práctica frecuente dentro de la estadística y las ciencias del comportamiento confundir el modelo con la teoría del modelo, o mejor, tomar a las suposiciones cuantitativas de la teoría, que en un tratamiento preciso serían los axiomas de la teoría, como el modelo. Los econometristas, anota Suppes, usan el término modelo para referirse a una clase de modelos, en el sentido lógico, mientras que llaman estructura a lo que los lógicos refieren por modelo (Cfr. Suppes, 1960: 111-112).

Presumiblemente, en conformidad con el concepto lógico de modelo, Moulines hace las siguientes acertadas observaciones sobre el sentido científico de modelo:

(a) no se supone que el 'modelo' cubra todos los aspectos del dominio empírico que describe, ni incluso todos aquellos considerados como importantes por alguna razón u otra; (b) no se supone que el 'modelo' provee la última verdad acerca del dominio en cuestión, sea lo que esto podría ser; [...] (c) la aceptación del 'modelo' permite sucesivas revisiones, refinamientos, supresiones, adiciones, etc., mientras que mantiene su "esencia" a través de esos cambios (2002: 1-2). 
En un estudio reciente sobre los modelos científicos, Daniela Bailer-Jones realizó una serie de entrevistas a científicos de distintos campos - astronomía, física nuclear, física de partículas elementales, física solar, física del estado sólido, química, biogeoquímica y paleontología evolucionista - preguntándoles qué son los modelos científicos, donde se puede constatar la polisemia del concepto de modelo en las disciplinas científicas actuales. Ella resume unas conclusiones extraídas de las entrevistas, de la siguiente manera:

Se reconoce que los modelos son centrales en la empresa científica actual.

Las teorías tienden a ser más fundamentales y más generales que los modelos, mientras que los modelos pueden extraerse de las teorías y así se muestra cómo las teorías contribuyen a modelar fenómenos específicos.

Los modelos simplifican las cosas y de ahí tratan de capturar la esencia de algo, mientras que dejan fuera detalles menos esenciales acerca del fenómeno modelado.

La validez de los modelos puede ser limitada, lo que significa que diferentes modelos pueden cumplir funciones diferentes. Así los modelos pueden ser útiles sin que se conozca que son "correctos" o incluso cuando se sabe que no son correctos.

Los modelos no sólo deben encajar con los datos empíricos disponibles, sino también deben dar lugar a predicciones y de esta manera ser contrastables (Bailer-Jones, 2009: 13).

Esta variedad de usos del término modelo, o quizá variedad de conceptos de modelo, ha dado lugar a que en la filosofía de la ciencia se hayan propuesto recientemente diversas tesis sobre los modelos en ciencia, destacando uno u otro aspecto de la construcción de modelos, de su estatus ontológico, de las funciones que cumplen, de sus relaciones con las teorías, por un lado, y con la experiencia científica y el mundo físico, por el otro. 
Se ha sostenido, por ejemplo, que los modelos son relativamente independientes de las teorías. Cartwright (1983) mantiene que hay modelos que concuerdan con ciertas leyes fenoménicas que se extraen de los fenómenos, las cuales no son derivables de las leyes fundamentales de las teorías, y que hay modelos incompatibles de un mismo fenómeno igualmente "correctos" (right) que estudian aspectos diferentes de éste.

Mary Morgan y Margaret Morrison (1999) han propuesto la tesis, en ese mismo tenor, de que los modelos son "agentes autónomos" intermediarios entre la teoría y el mundo. El uso lingüístico que ellas hacen del término modelo es simplemente el uso equívoco que se encuentra en los textos científicos, donde por igual se habla del modelo del átomo de hidrógeno de Bohr, del modelo de la doble hélice del ADN, del modelo de la superconductividad de London, del modelo de la Gran Explosión o del modelo estándar de la física de partículas elementales. En otras palabras, ellas consideran modelo todo aquello que los científicos nombran con ese término, como en los anteriores ejemplos, con posibles excepciones como la teoría física de las partículas elementales.

Sin embargo, esas autoras -y otros, como Ronald Giere (1988) - no caracterizan matemática, o formalmente lo que podrían ser esa variedad de modelos, dejando sin aclarar el estatus ontológico de los modelos, esto es, qué son los modelos; con lo cual se torna más complicado discernir las relaciones que pudieran guardar esas difusas entidades con las entidades y procesos que son objetos de estudio de la física.

Hay un problema más de fondo en la filosofía de la ciencia de Cartwright, que consiste simplemente en que ella no tiene una tesis sustancial sobre la relación de los modelos con los fenómenos o procesos físicos a los que se intentan aplicar. Como muchos otros autores, Cartwright apela a una figura, a una imagen: que los modelos asemejan (resemblance) a los procesos físicos concretos - lo 
que supondría algún tipo de semejanza entre entidades abstractas y objetos concretos. Esto es patente en trabajos posteriores de Cartwright, donde distingue entre dos tipos de modelos: por un lado, modelos interpretativos, que son los modelos anteriormente referidos en los que las leyes de las teorías resultan verdaderas; por otro lado, modelos representativos, "modelos que construimos con la ayuda de una teoría para representar arreglos y cosas que tienen lugar en el mundo —o podrían hacerlo bajo las circunstancias apropiadas" (1999: 242).

Mas como Bailer-Jones anota, Cartwright:

[...] no elabora el concepto de representación que usa para decir que las teorías no representan el mundo y que los modelos representativos lo hacen. Ella no quiere pensar a la representación como isomorfismo estructural. De acuerdo con Cartwright, la noción necesita ser más amplia que alguna 'basada en la simple idea de retratar [picturing]'. En su lugar sugiere 'una vaga noción de semejanza [resemblance]' (2008: 25). ${ }^{4}$

Así, la noción invocada de representación como semejanza, es tan indefinida y carente de precisión que más bien presenta un problema en vez de contribuir a resolver otro.

\section{El carácter idealizado y abstracto de los modelos físicos}

Bailer-Jones propone un concepto general de modelo en los siguientes términos: "Un modelo es una descripción interpretativa de un fenómeno que facilita el acceso al fenómeno ('fenómeno' refiere a 'cosas que acontecen')", explicando que "Las interpretaciones descriptivas pueden descansar, por ejemplo, en idealizaciones

${ }^{4}$ La propia Cartwright tácitamente ha reconocido esta observación crítica. Véase también la crítica a la posición de Cartwright en Hoefer (2008). 
o simplificaciones o en analogías con descripciones interpretativas de otros fenómenos. Facilitar acceso usualmente envuelve concentrarse sobre aspectos específicos de un fenómeno, algunas veces desatendiendo otros. Como resultado de ello, los modelos suelen ser sólo descripciones parciales" (2009: 1-2). Los modelos físicos abarcan, para Bailer-Jones, desde objetos materiales (como la maqueta de un aeroplano) hasta entidades teóricas, abstractas, como el modelo estándar de la estructura de la materia y sus componentes elementales.

Este concepto de modelo de Bailer-Jones acierta en destacar algunos rasgos característicos de los modelos en física, que abajo anoto para elaborarlos después por cuenta propia: Los modelos 1) consisten en interpretaciones de fenómenos o cosas que acontecen; 2) involucran idealizaciones o simplificaciones; 3) también involucran la selección de unos aspectos del fenómeno y la omisión de otros.

La primera cuestión apunta a que si bien los modelos, de alguna manera, describen los fenómenos o procesos físicos, lo hacen de manera interpretativa. ${ }^{5}$ Esto significa, en mi parecer, que las descripciones que proveen los modelos de los fenómenos no pretenden ser fidedignas al fenómeno; más bien, las descripciones se hacen interpretando al fenómeno desde una teoría o marco conceptual, precisamente a través del modelo.

$\mathrm{Al}$ decir lo anterior, queremos oponer interpretación a representación. Este último término connota ideas como semejar o simular, ideas que han desarrollado algunos autores como Giere y Cartwright, pero que se han mostrado implausibles en conexión con los modelos. Más bien la idea que queremos capturar es que los modelos son interpretaciones de lo que acontece en el mundo

\footnotetext{
${ }^{5}$ Bailer-Jones señala una variedad de medios de descripción, desde cosas concretas hasta entidades abstractas: textos lingüísticos, esquemas, diagramas, grafos y ecuaciones matemáticas (Cfr. 2009: 1-2).
} 
físico desde una teoría o marco conceptual, de tal suerte que, a lo sumo, los modelos resultan ser presentaciones de sistemas físicos desde la perspectiva de un marco teórico.

Tal vez lo anterior sea consistente con el concepto de modelo de Bailer-Jones, quien considera que la mayoría de los modelos científicos son altamente teóricos: "Ellos descansan a menudo en ideas y conceptos abstractos, frecuentemente empleando un formalismo matemático (como el modelo de la gran explosión, por ejemplo), pero siempre con la intención de proveer acceso a aspectos del fenómeno que se consideran esenciales" (Bailer-Jones, 2009: 2).

Los otros dos rasgos apuntan, respectivamente, al carácter idealizado y abstracto de los modelos en física. Sobre el concepto de idealización, Ernan McMullin señala que:

El término 'idealización’ es difuso. Lo tomaré para significar una simplificación deliberada de algo complicado (una situación, un concepto, etc.) con el fin de lograr al menos una comprensión parcial de esa cosa. Puede involucrar una distorsión del original o simplemente dejar a un lado algunos componentes de un complejo para concentrarse mejor en los demás (1985: 248).

El concepto de abstracción refiere a un proceso de selección de algunas magnitudes y parámetros relevantes para, digamos, explicar un género de procesos físicos, porque uno puede considerarlos como factores causales o influyentes, u otra razón explicativa. En verdad se trata del concepto aristotélico de abstracción, sobre el cual Cartwright escribe:

[...] Quisiera reservar la palabra 'abstracción' para una noción aristotélica, donde 'abstracción' significa 'eliminar' o 'substraer'. Para Aristóteles, empezamos con un particular concreto completo con todas sus propiedades. Después eliminamos — en nuestra imaginación - todo lo que es irrelevante para nuestros propósitos del momento para concentrarnos en una única propiedad o con- 
junto de propiedades, 'como si ellas estuvieran separadas' (1989: 197).

McMullin no separa claramente los conceptos de idealización y abstracción, como podemos constatar en el pasaje recién citado, donde él escribe que la idealización galilieana "puede involucrar una distorsión del original o puede simplemente dejar a un lado algunos componentes de un complejo [...]". Para ver esto, consideremos el ejemplo paradigmático de modelo físico: el modelo del átomo de hidrógeno de Bohr.

McMullin anota que, originalmente, Bohr hizo tres simplificaciones irrealistas: 1) que el núcleo del átomo permanece en reposo, 2) que la órbita del electrón es circular, y 3) una omisión de los efectos relativistas debidos al rápido movimiento del electrón. Posteriormente esas suposiciones fueron removidas de ese modelo, introduciendo: “[...] el pequeńo movimiento del núcleo alrededor del centro común de gravedad del protón y el electrón produciendo un factor de corrección de $(1+m / M)$ en la fórmula espectral, donde $m / M$ es la razón de las masas del electrón y el protón”, órbitas elípticas y una corrección de la fórmula de las series para dar cuenta de la dependencia relativista de la masa del electrón de su velocidad que desvía las series enteras por una cantidad muy pequeña (1985: 260-261).

Podemos percatarnos de que en el modelo original de Bohr las dos primeras suposiciones son idealizaciones, en el que sentido de que involucran una distorsión del sistema, mientras que la tercera es, más bien, una omisión de un factor, y así el resultado de un proceso de abstracción. Aunque las tres suposiciones simplifican el sistema, podemos decir que las dos primeras son contrafácticas, mientras que la última es sólo contra-actual (contractual). De esta manera la noción de simplificación de un sistema físico, sea deliberada o no, es insuficiente. Se precisa de las nociones de distorsión y selección (de ahí, exclusión) que están comprimidas en aquélla. Y 
como este mismo ejemplo muestra, la desidealización del modelo de Bohr envuelve tanto eliminar una suposición irrealista como agregar un factor de corrección.

Por razones de claridad, diremos que tanto en la idealización como en la abstracción están involucradas algunas suposiciones irrealistas. En el primer caso, las suposiciones son más bien contrafácticas, mientras que en el segundo son suposiciones contraactuales. Podemos decir, con respecto a una formulación más explícita de una ecuación o enunciado nómico asociado a un modelo físico, que i) si la suposición irrealista es contrafáctica (una distorsión) entonces la adición de un factor de corrección es un proceso de desidealización, y ii) si la suposición irrealista es contractual (una omisión) entonces la adición de un factor de corrección es un proceso de concretización.

De nuevo, en el ejemplo del modelo de Bohr la remoción de la suposición contrafáctica de que el núcleo del átomo permanece en reposo y la correspondiente introducción del factor de corrección en la fórmula espectral es un proceso de desidealización, mientras que introducir una corrección en la fórmula de series para dar cuenta de los efectos relativistas es una suposición contra-actual, es un proceso de concretización. ${ }^{6}$

Hemos señalado los procesos intelectuales de idealización y abstracción que entran en juego en la construcción de un modelo físico (y los respectivos procesos inversos requeridos para mejorar el modelo y poder aplicarlo a sistemas físicos). Estos procesos son propios y característicos de la teorización en el campo de la física, y se precisan para obtener modelos que nos permitan conceptualizar los fenómenos o procesos que sufren los sistemas físicos. Además,

${ }^{6}$ Sin embargo, en la medida en que una omisión puede ser considera no sólo como una simplificación sino también como una distorsión o deformación del sistema (p. ej., sistemas libres de fricción o de efectos relativistas), esta distinción sería insuficiente porque los dos conceptos se superpondrían en tales casos. 
los modelos son parciales, como señalan Moulines y Bailer-Jones, y están sujetos a revisión y modificación, como anota Moulines.

En su modelo original, Bohr asumió, de manera no deliberada, que el electrón tiene una trayectoria clásica definida en estados estacionarios. Actualmente esta suposición resulta "irrealista" porque las descripciones que la teoría cuántica da de esas trayectorias son en términos de funciones de probabilidad de densidad alrededor del núcleo. Esto muestra que no todas las suposiciones simplificadoras incorporadas en un modelo - en alguna etapa del desarrollo de una teoría física-pueden ser eliminadas por uno de los dos procesos inversos seńalados, sino que se requiere más bien de reconceptualizar el sistema en cuestión bajo diferentes suposiciones, que involucran una nueva idealización del sistema.

Por los anteriores procesos inversos, sea desidealización o concretización, las formulaciones de las leyes de una teoría pueden mejorarse en el sentido de que se aumenta su contenido físico, se suprimen algunas suposiciones irrealistas y se incorporan más factores relevantes. El resultado es enunciados nómicos más realistas, concretos y específicos, que dan lugar a construir modelos asociados que sean, a su vez, más realistas, concretos y específicos, siendo los candidatos a ser aplicados a los sistemas físicos individuales.

Tales modelos proveen de conceptualizaciones de sistemas físicos dentro del marco de una teoría, mientras que las leyes asociadas pueden ser aplicadas para comprender y, eventualmente, explicar lo que acontece en esos sistemas. En la medida en que un modelo pueda ser desidealizado, su aplicación a sistemas físicos resulta más realista. Esto implica que la capacidad descriptiva de un modelo físico se incrementa en la medida en que el modelo sea desidealizado, lo cual contribuye a que nuestra interpretación del fenómeno o proceso al que aplicamos el modelo sea más concreta y específica, mejorando nuestra comprensión de éste. 


\section{Conclusión}

La variedad de los modelos construidos por los físicos hace compleja la tarea de caracterizarlos de manera general. Si bien podemos descartar, por un lado, los modelos materiales a escala por ser ininteresantes y, por el otro, el modelo estándar de las partículas elementales, ya que realmente es un enjambre de teorías sobre la materia y las interacciones entre sus componentes, hay modelos físicos como los modelos de las partículas elementales, el modelo de la doble hélice del ADN y el modelo de la superconductividad de London que difícilmente podríamos capturar en un único concepto general.

El concepto de modelo propuesto por Bailer-Jones tiene la virtud, en contraste con otros conceptos, de caracterizar los modelos no como representaciones sino como interpretaciones y de reconocer, implícitamente, el carácter idealizado y abstracto de los modelos.

Desde una posición conceptualista, hemos argüido que las interpretaciones de los fenómenos o procesos físicos de las que nos proveen los modelos, son presentaciones de tales entidades desde la perspectiva de un marco teórico. Esto da cabida a tener modelos alternativos, incluso incompatibles, como Cartwright y sus colegas han señalado, que capturen distintos aspectos de un mismo fenómeno bajo distintas suposiciones teóricas.

Si bien puede argüirse que se han construido, y se construyen, modelos sobre ciertos géneros de fenómenos o procesos físicos sin que se tenga una teoría propiamente dicha de ellos - que es el caso del modelo original de Bohr-, como esas filósofas lo han hecho, podemos percatarnos que, incluso en esos casos, en el contexto del desarrollo de una teoría los modelos descansan sobre algunas hipótesis o suposiciones teóricas, que si se articulan conforman una 
incipiente teoría, parcial y aproximada, del fenómeno o proceso en cuestión.

Podemos decir que si se explicitan y se articulan todos los supuestos tácitos junto con las aserciones expresas del modelo original de Bohr, se obtiene una incipiente teoría del átomo de hidrógeno. Y agregar que el desarrollo de la teoría de la materia y las interacciones de sus elementos ha consistido, en parte, en avanzar postulaciones y elaboraciones teóricas, así como en la construcción de modelos de las partículas elementales actualmente reconocidas.

La tesis de Suppes es que, en principio, podemos tomar a las suposiciones cuantitativas de una teoría para formularla axiomáticamente y delimitar, así, la clase de sus modelos. Nosotros hemos intentado enfatizar que tanto las suposiciones implícitas como las hipótesis propuestas explícitamente — sea en la construcción de un modelo o en la formulación de una teoría - involucran la abstracción de ciertas propiedades cuantificables del sistema físico bajo estudio y la idealización de las entidades físicas que lo conforman.

\section{Bibliografía}

Bailer-Jones, Daniela M., 2008, "Standing Up Against Tradition Models and Theories in Nancy Cartwright's Philosophy of Science" en S. Hartmann, C. Hoefer y L. Bovens (eds.), Nancy Cartwright's Philosophy of Science, Nueva York / Londres, Routledge Studies in the Philosophy of Science, pp. 17-37.

, 2009, Scientific Models in Philosophy of Science, Pittsburgh, University of Pittsburgh Press.

Balzer, W., U. Moulines y J. Sneed, 1987, An Architectonic for Science, Dordrecht, Reidel.

Cartwright, N., 1983, How the Laws of Physics Lie, Oxford, Clarendon Press. 
, 1989, Nature's Capacities and their Measurement, Oxford, Clarendon Press.

Giere, Ronald, 1988, Explaining Science; A Cognitive Approach, Chicago, University of Chicago Press.

Hoefer, C., 2008, "For Fundamentalism" en S. Hartmann, C. Hoefer y L. Bovens (eds.), Nancy Cartwright's Philosophy of Science, Nueva York / Londres, Routledge Studies in the Philosophy of Science, pp. 307-321.

Hughes, R. I. G., 1997, "Models and Representation”, Philosophy of Science, vol. 64, pp. 325-336.

Liu, Chuang, 2004, "Laws and Models in a Theory of Idealization", Synthese, vol. 138, pp. 363-385.

McMullin, Ernan, 1985, "Galilean Idealization”, Studies in the History and Philosophy of Science, vol. 16, núm. 3, pp. 247-273.

Morrison, M. y M. Morgan, 1999, "Models as Mediating Instruments" en M. Morrison y M. Morgan (eds.), Models as Mediators, Cambridge, Cambridge University Press, pp. 10-37.

Moulines, C. Ulises, 2002, "Introduction: Structuralism as a Program for Modelling Theoretical Science”, Synthese, vol. 130, pp. $1-11$.

, 2008, El desarrollo moderno de la filosofía de la ciencia (1890-2000), Xavier de Donato (trad.), México, UNAM, 2011.

Muller, F. A., 2011, "Reflections on the Revolution at Stanford", Synthese, vol. 183, pp. 87-114.

Redhead, Michael, 1980, "Models in Physics", British Journal for the Philosophy of Science, vol. 31, núm. 2, pp. 145-163.

Sneed, Joseph, 1971, The Logical Structure of Mathematical Physics, Dordrecht, Reidel. 
Suppes, Patrick, 1953, "Filosofía y los fundamentos axiomáticos de la física" en Estudios de filosofía y metodología de la ciencia, José Luis Rolleri (trad.), Madrid, Alianza Universidad, 1988, pp. 21-27. , 1957, Introduction to Logic, Nueva York, Van Nostrand. , 1960, "Una comparación del significado y los usos de los modelos en las matemáticas y las ciencias empíricas" en Estudios de filosofía y metodología de la ciencia, Mario Alberto Cortez Rodríguez (trad.), Madrid, Alianza Universidad, 1988, pp. 109-123.

Tarski, Alfred, 1953, "A General Method in Proofs of Undecidability" en A. Tarski, A. Mostowski y R. M. Robinson (eds.), Undecidable Theories, Amsterdam, North-Holland.

Van Fraassen, Bas, 1980, La imagen científica, Sergio Martínez (trad.), México, Paidós / UNAM, 1996.

(Artículo aceptado el 20 de mayo de 2013) 